The FASEB Journal express article 10.1096/fj.04-2210fje. Published online December 29, 2004.

\title{
A comparison of strain and fluid shear stress in stimulating bone cell responses - a computational and experimental study
}

\author{
James G. McGarry,* Jenneke Klein-Nulend, ${ }^{\dagger}$ Margriet G. Mullender, ${ }^{\dagger}$ and
}

Patrick J. Prendergast*

*Centre for Bioengineering, Department of Mechanical Engineering, Trinity College, Dublin, Ireland; ${ }^{\dagger}$ Department of Oral Cell Biology, ACTA-Universiteit van Amsterdam and Vrije Universiteit, Amsterdam, The Netherlands.

Corresponding author: Patrick Prendergast, Centre for Bioengineering, Department of Mechanical Engineering, Trinity College, Dublin 2, Ireland. E-mail: pprender@tcd.ie; www.biomechanics.ie

\section{ABSTRACT}

Bone undergoes continuous remodeling in response to mechanical loading. However, the underlying mechanisms by which bone cells respond to their changing mechanical environment, that is, strain in the load-bearing matrix or fluid flow through the canalicular network, are not well understood. It has been established in vitro that bone cells respond differently to substrate strain and fluid shear stress treatments. Uncovering the mechanical basis of these differences represents a significant challenge to our understanding of cellular mechanotransduction and bone remodeling. To investigate this problem, we developed a biomechanical model of an adherent cell, to test the hypothesis that bone cells respond differently to $0.6 \mathrm{~Pa}$ fluid shear stress and $1,000 \mu \varepsilon$ substrate strain stimulation because of qualitative and quantitative differences in the cellular deformation caused. Fluid shear stress loading conditions resulted in maximum displacements at the apical surface of the cell $\sim 8$ times higher than those due to strain at the cellsubstrate interface and also caused higher stressing of all parts of the cell. Significantly, this shows that the deforming effects of fluid shear stress and strain on a cellular level are qualitatively different, which may provide a basis for explaining differences in bone cell responses to both stimuli as reported in several studies. Although our approach to modeling the morphology and complex physical environment of an adherent cell is certainly simplified, our results do show independent roles for fluid flow and strain as mechanical stimuli and highlight the importance of deformation on a cellular level in bone physiology.

Key words: cell deformation • cell biomechanics • bone remodeling

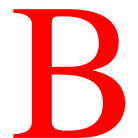
one tissue is adapted in a continuous sequence of bone remodeling in response to musculoskeletal loading $(1,2)$. Although the remodeling process involves the formation of bone by osteoblasts in areas excavated by osteoclasts, the mechanisms involved in cell activation as a result of mechanical loading are poorly understood. Possible stimuli include cell 
strain as a result of deformation in the load-bearing matrix (3) and strain-induced fluid flow through the lacuno-canalicular network (4-6). To examine the effect of strain and fluid flow on cells, a variety of apparatuses have been used to apply strain and fluid shear stress to bone cells in culture (7). Bone cells have been found to respond to fluid shear stress with increases in intracellular calcium concentration (8) and the release of signaling molecules (9-12), while strain has been found to increase the production of matrix proteins (13) and collagen type I (14), cell proliferation (15), and $c$-fos gene expression (16). Studies in which bone cell responses to both fluid shear stress and straining treatments have been directly compared also report large differences in responsiveness to both mechanical stimuli (17-19). Revealing why bone cells should respond differently to these two kinds of mechanical stimulation represents a significant challenge to our understanding of cellular mechanotransduction in mechanobiology. In particular, we would like to know if there are any qualitative and quantitative differences in cell deformation under the magnitudes of stimulation used in in vitro experiments.

Previous approaches for computational modeling of cells have treated the cytoplasm and cytoskeleton as a continuum. Examples of this approach include models of suspended cells comprising a cortical membrane and viscous cytoplasm (20), a chondrocyte embedded in its extracellular matrix (21), layers of airway epithelial cells (22), and a three-dimensional model comprising a membrane/cortex and viscoelastic cytosol (23). However, a growing number of studies have highlighted the importance of the cytoskeleton in providing structural stability in adherent cells and in transmitting mechanical loads from the cell surface through the cytoplasm into the nucleus $(24,25)$. For this reason, the cytoskeleton has been modeled as a percolation network of fibers in a viscous cytoplasm (26). Other similar approaches that model the cytoskeleton explicitly include the tensegrity approach. In this approach the cytoskeleton is considered to be a stress-supported network of interconnected filaments and tubules $(27,28)$. By attributing a central role to cytoskeletal contractile forces (prestress), the tensegrity approach has described several aspects of cellular structural behavior (29). It is our opinion, however, that a cell model combining many structurally significant cellular components in an idealized morphology, to which mechanical loads could be applied, would be a valuable tool in investigating the deforming effect of fluid shear stress and strain stimulation on a cellular level.

In the present paper, we describe an experiment in which we measured the NO, PGE 2 , and collagen type I response in human bone cells to fluid shear stress of magnitude $0.6 \mathrm{~Pa}$ and substrate strain of magnitude $1,000 \mu \varepsilon$ using identical culturing protocols, such that cellular responses to the two mechanical loading treatments could be directly compared. We tested the hypothesis that differences in bone cell response to both magnitudes of fluid shear stress and strain can be explained by the amount of cellular deformation caused. For this purpose, we developed a computational model of a cell adherent on a substrate comprising cytoskeletal, nucleus, cytoplasm, and membrane components, and used this model to compute cellular deformation under fluid shear stress and substrate strain-loading conditions. By relating deformation computed by the cell model to our and other published experimental findings, we aim to propose why $\mathrm{NO}$ and $\mathrm{PGE}_{2}$ release and collagen type I production should be so different due to both mechanical stimuli and discuss the implications of our findings for understanding cellular mechanotransduction and bone remodeling. 


\section{METHODS}

\section{Experimental protocol}

\section{Treatment of bone cells with fluid shear stress and substrate strain}

We obtained primary human bone cells from bone fragments of 3 donors (2 males, 14 and 16 years; 1 female, 11 years) who received surgery after trauma. Human bone cells were prepared from small bone fragments, which were placed in $25 \mathrm{~cm}^{2}$ tissue culture flasks (Sarstedt, Germany). The outgrowing bone cells were cultured in $\alpha$-MEM, supplemented with $10 \%$ fetal bovine serum (FBS; Sigma-Aldrich), $50 \mu \mathrm{g} / \mathrm{ml}$ of ascorbic acid, $10 \mathrm{mM}$ of $\beta$-glycerophosphate and 1\% antibiotic-antimycotic solution (Sigma, St. Louis, MO). The cells were grown over two passages and were then plated, two days before each experiment, at $5 \times 10^{5}$ cells on polylysinecoated glass slides. Cells were cultured for $24 \mathrm{~h}$ in $\alpha$-MEM containing $10 \%$ FBS, antibiotics and ascorbate, as described above. The amount of FBS was then reduced to $2 \%$ for $24 \mathrm{~h}$ incubation, and the subconfluent layers of cells were subsequently loaded either with fluid shear stress (0.6 \pm $0.3 \mathrm{~Pa}, 5 \mathrm{~Hz}$ ) for $1 \mathrm{~h}$ in a parallel plate chamber, as described previously (9), or with substrate strain $(1,000 \mu \varepsilon, 1 \mathrm{~Hz})$ for $1 \mathrm{~h}$ using a four-point bending apparatus (16). Untreated controls were kept in petri dishes containing $13 \mathrm{ml}$ medium under similar conditions as the experimental cultures, that is, at $37^{\circ} \mathrm{C}$ in a humidified atmosphere of $5 \% \mathrm{CO}_{2}$ in air. The release of nitric oxide (NO) was measured as nitrite accumulated in the conditioned medium using Griess reagent, and prostaglandin $\mathrm{E}_{2}\left(\mathrm{PGE}_{2}\right)$ was measured using an enzyme immunoassay, as described previously (12). The amount of collagen type I produced was measured by Western blot analysis. Comparisons between treated and control cultures were made using either the Wilcoxon signed rank test ( $\mathrm{NO}$ and $\mathrm{PGE}_{2}$ ) or the general linear model (collagen type I).

\section{Computational cell model}

\section{Finite element method}

The finite element method is a mathematical tool that can be used to calculate the deformation of three-dimensional shapes divided into a finite number of elements and nodes under any desired loading conditions. The method has proven a useful tool for insight into mechanobiology at the microscopic level where experimental measurements are often difficult (30). In the present study the finite element method was used to develop a computational model of an adherent cell and to compute the deformation of a single cell as a result of fluid shear stress and substrate strain loading conditions. Cellular deformation was expressed as the displacement $(\mu \mathrm{m})$ of the cell model, or as the von Mises stress induced in the various model components, relative to original material properties (\% deformation). Von Mises stress $\left(\sigma_{e}, \mathrm{~N} . \mu \mathrm{m}^{-2}\right)$ combines the stress values in each direction:

$$
\sigma_{\mathrm{e}}=\left\{\frac{1}{2}\left[\left(\sigma_{\mathrm{xx}}-\sigma_{\mathrm{yy}}\right)^{2}+\left(\sigma_{\mathrm{xx}}-\sigma_{\mathrm{zz}}\right)^{2}+\left(\sigma_{\mathrm{yy}}-\sigma_{\mathrm{zz}}\right)^{2}+6\left(\sigma_{\mathrm{xx}}^{2}+\sigma_{\mathrm{xz}}^{2}+\sigma_{\mathrm{yz}}^{2}\right)^{2}\right]\right\}^{1 / 2} .
$$

The cell model geometry, the material properties for each model component, and the loading conditions used to model both fluid shear stress and strain are described below. The finite element software ANSYS 7.0 (Canonsburg, PA, USA) was used. 


\section{Cell model geometry}

On the basis of images of a spreading cell (ig. 1), a finite element model was developed to represent a spread bone cell adherent on a flat substrate (Fig. 2). This idealized biomechanical model comprises a nucleus, an internal cytoskeleton of tensile actin and compressive microtubule elements, together with cytoplasm, and membrane components (Fig. 2). The nucleus was formed from an ellipse with a major axis of $8 \mu \mathrm{m}$, minor axis of $5 \mu \mathrm{m}$, and a distance of 2 $\mu \mathrm{m}$ from the lower flat surface of the model representing the cell-substrate interface (Fig. 2). The cytoplasm and nucleus components are meshed with 8-node lower-order hexahedral elements, and the membrane is meshed with four-node shell elements (Fig. 2). Microtubule struts and microfilament cables are meshed with single link elements (2-node three-dimensional spar elements with a bilinear stiffness matrix), supporting compression-only and tension-only respectively (Fig. 2). The end of each strut connects with four cables at 12 common nodes, which are also coincident with membrane and underlying cytoplasm nodes, and are therefore analogous to "receptor" sites or focal adhesion complexes (Fig. 2). This coincidence of nodes is achieved through either resizing of the solid cytoplasm (and membrane shell) elements, or by slight repositioning of nodes of the cytoskeletal elements.

The internal cytoskeleton in the model was formed by repositioning an originally rounded tensegrity structure (Fig. 3) to comply with the more spread shape of the model (Fig. 1 and 2 ). This was achieved by establishing new positions for nine cytoskeletal receptor nodes (nodes 4 to 12 in Fig. 3) on the membrane surface of the model. To determine the exact new receptor positions, radial planes defined by the central axis of the model and the original positions of the receptor nodes in Fig. 3 were established. The intersection of these radial planes with the model surface (Fig. 2) yielded lines along which the new nodal positions must lie. Points of intersection of these surface lines with horizontal planes that maintain the vertical proportions of the original tensegrity structure, that is, the planes defined by receptor nodes 4, 5, 6, nodes 7, 8, 9 and nodes 10, 11, 12 (Fig. 3), yielded new positions for cytoskeletal receptor nodes on the membrane surface (Fig. 2). The original lengths and positioning of microtubule and microfilament elements are altered to comply with these new receptor positions to yield the configuration for the internal cytoskeleton in the biomechanical model (Fig. 2). The cell-substrate interface of the model (Fig. 2) includes three end nodes (nodes 1, 2, 3 in Fig. 3) of microtubule struts, one from each orthogonal direction.

\section{Constitutive modeling of cellular components}

Material properties for cellular components of bone cells are currently unknown and were therefore estimated from published data for various cell types (Table 1). The cytoplasm and nucleus were treated here as linear elastic and isotropic continua. Following Kamm et al. (22), the elastic modulus of the cytoplasm is chosen as $100 \mathrm{~Pa}$. The nucleus has been reported as 4 times stiffer than the cytoplasm (32) and is therefore taken as $400 \mathrm{~Pa}$. Poisson's ratio ( $v$ ) for both nucleus and cytoplasm is taken to be 0.37 (33). In a model developed by Kamm et al. (22), in which epithelial cell membrane elasticity was specified as $10^{7} \mathrm{~Pa}$ with a thickness of $6 \mathrm{~nm}$, it was concluded that membrane elasticity was likely overestimated. In the model a lower value of $10^{3}$ $\mathrm{Pa}$ was therefore taken (with $v=0.3$ ), while maintaining the same membrane thickness. The elastic properties applied to the cytoskeletal components in the model are based on the elasticity values determined by Gittes et al. (34), who measured the flexural rigidity (bending stiffness) of 
microtubules and microfilaments when subjected to thermal fluctuations. In the model the microtubules were assigned cross-sectional areas of $190 \mathrm{~nm}^{2}$, while the microfilaments are thinner with an area of $18 \mathrm{~nm}^{2}$. These values were also taken from the study by Gittes et al. (34).

\section{Application of fluid shear stress and strain loading conditions to the cell model}

\section{Boundary conditions}

In our experiments, substrate strain and fluid shear stress treatment were applied to monolayers of subconfluent cells. In our modeling approach, it was therefore assumed that each cell was attached only to the underlying substrate and not to neighboring cells. This assumption allows us to compute the cellular deformation occurring as a result of fluid shear stress and strain treatment using a model of a single cell (Fig. 2). Cytoskeleton nodes 1, 2, 3 and membrane nodes on the lower surface (cell-substrate interface) of the model were constrained in all degrees of freedom and are therefore analogous to focal adhesion sites in adherent cells. No initial strain (prestress) was applied to either microfilaments or microtubules, although microfilament prestress was subsequently varied to determine its impact on model results.

As the material properties assigned to cell model components are linear elastic and because geometric nonlinearities can be neglected given the very small displacements occurring, by computing the deforming effect of one magnitude of fluid shear stress or substrate strain, we can directly infer the deformations that would occur at various magnitudes of fluid shear stress or strain as used in other published studies.

\section{Loading conditions to model substrate strain}

We used the four-point bending apparatus; because the height of the adherent bone cells $(<7$ $\mu \mathrm{m})$ is small relative to the thickness of the bending substrate $(200 \mu \mathrm{m})$, the bone cells were subjected to axial strain only and not to bending moments. This axial strain was calculated and subsequently confirmed by strain gauge measurements as 1,000 $\mu \varepsilon$. To model the effect of this strain using the cell model, each node at the cell-substrate interface was displaced axially by 0.1\% $(1,000 \mu \varepsilon)$ strain (Fig. 4A). As Poisson's ratio $(v)$ for the glass substrate equals 0.3, the corresponding displacement of nodes in the lateral direction was taken as $0.03 \%$ strain (Fig. $4 A$ ).

\section{Loading conditions to model fluid shear stress}

As the fluid flow in the parallel plate chamber (12) was laminar, it was assumed that the shear stress to which the cells were subjected was equal to the theoretical shear stress at the wall (calculated as $0.6 \mathrm{~Pa})$. Recognizing that cellular dimensions $(<7 \mu \mathrm{m})$ are small relative to the distance between the plates $(\sim 300 \mu \mathrm{m})$, it was assumed that this shear stress acts tangentially along the cell surface in the direction of flow. The forces applied to the cell model were found from the shear stress, denoted $\tau$, distributed over the surface area exposed to fluid flow, denoted $A_{S}$,

$$
F=\tau \times A_{S}
$$

From the model (Fig. 2) it can be found that $A_{S}=1329 \mu \mathrm{m}^{2}$, and so 


$$
\mathrm{F}=0.6 \mathrm{pN} / \mu \mathrm{m}^{2} \times 1329 \mu \mathrm{m}^{2}=797.4 \mathrm{pN}
$$

This force was assumed to distribute evenly over the membrane surface area. The total force applied to each node is the sum of one quarter of the force assigned to each of its surrounding elements. As it is assumed that shear stress acts tangentially, nodal forces are then resolved to horizontal and vertical components based on the contact angle between each node and the cellsubstrate horizontal plane (Fig. 4B).

\section{RESULTS}

We found large differences in bone cell responses to 0.6 Pa fluid shear stress compared with $1,000 \mu \varepsilon$ substrate strain treatment. After $60 \mathrm{~min}$, fluid shear stress caused a 7.1-fold increase in NO release and a 3.3-fold increase in $\mathrm{PGE}_{2}$ release, whereas substrate strain caused only 1.65fold and 1.3-fold increases in NO and $\mathrm{PGE}_{2}$ release, respectively (Fig. 5A). On the other hand, after $60 \mathrm{~min}$ and post-incubation for $24 \mathrm{~h}$, collagen I production was increased 1.6-fold by substrate strain, whereas it was decreased 0.6-fold by fluid shear stress (Fig. 5B).

The contour plots of displacements in the cell model due to both fluid shear stress and strainloading conditions illustrate that there are fundamental qualitative differences in the cellular deformation caused by both types of mechanical stimuli (Fig. 6). Fluid shear stress has a larger overturning effect on the bone cells, while the effect of substrate strain is focused on cellsubstrate attachments. These qualitative differences would also be predicted no matter what magnitude of stimulation was used. Maximum displacement due to $1,000 \mu \varepsilon$ substrate strain was computed as $19 \mathrm{~nm}$ at the cell-substrate interface, whereas 0.6 Pa fluid shear stress caused a much larger displacement of $106 \mathrm{~nm}$ along the apical surface where resistance to deformation is minimal (Fig. 6).

The stress values induced throughout the cell model reflect the extent of cellular deformation and hence stimulation caused by both fluid shear stress and strain. The deformation predicted in all cell components is given in Fig. 7. For example, the maximum stress induced in the membrane component is $\sim 7.5$-fold higher in fluid shear stress than in strain (Fig. 7). These maximum stress values occur at receptor sites due to the more rigid underlying cytoskeleton. Although deformation is smaller in magnitude in the more internal parts of the model, that is, the nucleus and cytoplasm-nucleus region, it is noteworthy that the differences in deformation between both methods of mechanical stimulation are greater in this region (Fig. 7A). This suggests that, in addition to causing higher deformation of all cellular components, fluid shear stress appears to transmit deformation more effectively to internal cellular regions than does substrate strain.

The cellular deformation that results from both mechanical stimuli can be related to the corresponding experimentally determined human bone cell responses to both fluid shear stress and substrate strain treatments; this involves correlating the results in Fig. 5 with those in Fig. 7 (see Discussion).

To investigate the stimuli under higher levels of substrate strain, we computed the strain that would yield a maximum membrane displacement equivalent to that caused by 0.6 Pa fluid shear stress. We found it to be $5,531 \mu \varepsilon$. The strain values required to induce equal stress in the 
nucleus were even higher $(\sim 8,200 \mu \varepsilon)$ since deformation is not distributed uniformly throughout the cell model components.

By varying the material properties of the various components of the cell model, it was found that cytoplasm elasticity has a large influence on cellular deformation. For example, increasing cytoplasm elasticity by a factor of 2 (to $200 \mathrm{~Pa}$ ) reduces nucleus deformation as a result of fluid shear stress from the original $0.48 \%$ to $0.34 \%$. Decreasing cytoplasm elasticity to $50 \mathrm{~Pa}$ increases nucleus deformation to $0.6 \%$. Variations in cytoplasm elasticity have less of an influence on cellular deformation under substrate strain-loading conditions, again indicating the more effective transmission of deformation in fluid shear stress. Increases in microtubule elasticity, or introducing prestress (initial strain values $>0$ ) to the microtubule or microfilament elements, resulted in increased membrane and cytoplasm stresses in the region of receptor sites, but did not alter the overall differences in deformation between fluid shear stress and strain-loading conditions found in the model. This is because maximum displacements occur at points of least resistance, that is, at a distance from the cytoskeleton.

\section{DISCUSSION}

When we experimentally stimulated bone cells with $0.6 \mathrm{~Pa}$ fluid shear stress and 1,000 $\mu \varepsilon$ substrate strain, we found large differences in the release of $\mathrm{NO}$ and $\mathrm{PGE}_{2}$ signaling molecules and in the production of collagen type I. When the experiment was analyzed with a computational cell model it was revealed that fluid shear stress of magnitude 0.6 Pa and substrate strain of magnitude $1,000 \mu \varepsilon$ are quite different in terms of the pattern of cellular deformation that they cause. Fluid shear stress causes significantly higher displacement and stressing of all cell model components (i.e., cytoplasm, cytoskeleton, nucleus, and membrane) relative to strain. The differences in displacements and stressing predicted as a result of fluid shear stress and strain at these magnitudes are important in investigating the origins of these differences in cell responses found in our experiments (Fig. 5), but it also explains the results of other studies in which cellular responses to similar magnitudes of fluid shear stress and strain were also compared $(18,19)$.

For example, we can explain that fluid shear stress, but not mechanical strain, induces increased $\mathrm{PGE}_{2}$ release (reported here and in (18)) because it causes higher displacement and stressing of the membrane and cytoskeleton. It has been shown that 1) flow-induced PGE $E_{2}$ release by bone cells is dependent on the cytoskeleton (35); and 2) fluid shear-induced increases in COX-2 expression (COX-2 is a key enzyme for $\mathrm{PGE}_{2}$ production) involve reorganization of the actin cytoskeleton (36). Therefore, it is very likely that increased PGE 2 activity may be related to higher displacement of the cytoskeleton in fluid shear stress as compared with strain. Similarly, the higher NO response due to fluid shear stress could be due to higher membrane stress because it has been suggested that mechanically induced formation of $\mathrm{NO}$ results from activation of endothelial cell nitric oxide synthase (ecNOS) in bone cells $(11,37,38)$. ecNOS is an enzyme bound to the plasma membrane that may be rendered susceptible to activation by increased stressing of the cell membrane (11). We chose $\mathrm{NO}$ and $\mathrm{PGE}_{2}$ as parameters of bone cell mechanosensitivity because 1 ) both $\mathrm{NO}$ and $\mathrm{PGE}_{2}$ have been shown to mediate bone formation in vivo, since inhibition of either pathway around the time of mechanical stimulation prevents osteogenic response (39-44) and 2) several previous studies have consistently reported that NO 
and $\mathrm{PGE}_{2}$ release are increased by fluid shear stress treatment over a range of time points (9-12, $18,35,45)$.

Our findings of increased cellular deformation in fluid shear stress can also shed some light on experimental reports by You et al. (19) of significant increases in $\mathrm{Ca}^{2+}$ due to oscillatory fluid flow treatment and not to physiological strain. These increases in $\mathrm{Ca}^{2+}$ may be caused by higher membrane stress in fluid flow, particularly since it is known that stimulation of the plasma membrane influences ion channel activity that can elicit rapid intracellular $\mathrm{Ca}^{2+}$ responses (46). Finally, regarding the opposite effects that fluid shear stress and strain have on collagen I production as reported here, the model can provide an explanation because it predicts that there are much higher stresses on the cell substrate attachments under substrate strain compared with fluid shear stress. This possible mechanism of stimulating collagen I production in bone cells could equally apply to the other studies that report increased activity related to matrix production in response to strain stimulation $(13,14)$.

We maintain that there is a biological basis for cell deformation when subjected to strain transmitted via the substrate because the frequency of applied strains $(1 \mathrm{~Hz})$ exceeds the rate at which new adhesion bonds can be formed. We contend that because strain-induced deformations have a basis, the fact that 0.6 Pa fluid shear stress causes even greater cellular deformation than $1,000 \mu \varepsilon$ substrate strain means that fluid shear stress deformations must also have a biological basis. Although, this remains to be confirmed for bone cells, images of rolling leukocytes deforming (decreased cell height and increased cell-substrate contact length) under fluid shear stresses ranging from 0.2 to $1.5 \mathrm{~Pa}$ (47) support our contention that bone cells deform under fluid shear stress also. In the present study, we report that cellular responses to fluid shear stress of magnitude 0.6 Pa and substrate strain of magnitude 1,000 $\mu \varepsilon$ should not be considered equivalent because the deformation occurring on the cellular level is not equal. However, since deformation of the cell model will vary linearly, we can extend our model results to interpret cellular responses to both fluid shear stress and strain stimuli of varying magnitudes. For example, we found that increasing the magnitude of strain in the computational model (to $\sim 5,500 \mu \varepsilon$ ) yields deformations in the range of those caused by fluid shear stress of magnitude 0.6 $\mathrm{Pa}$. If, as our results suggest, the release of $\mathrm{NO}$ and $\mathrm{PGE}_{2}$ signaling molecules is deformation-dependent, then such an increased magnitude of strain should result in equivalent cellular responses. In fact, this hypothesis is confirmed by several experimental findings. Higher NO release by bone cells has been reported at 3,400 $\mu \varepsilon$ (37), and 3,800 $\mu \varepsilon(48)$, and significant increases in $\mathrm{PGE}_{2}$ release by primary human osteoblasts at 4,000 $\mu \varepsilon$ (49). Essentially, this further strengthens our explanation that the preferential in vitro stimulation of $\mathrm{NO}$ and $\mathrm{PGE}_{2}$ release by fluid shear stress of magnitude $\sim 0.6 \mathrm{~Pa}$ is as a result of the higher deformation of both the membrane and the cytoskeleton.

Many in vitro studies, including ours, report that bone cells respond to strain with increased collagen or matrix production $(13,14)$ or with increased proliferation (15). Hence, applied strain induces cell behavior associated with the osteoblastic phenotype. This makes sense when we consider that the magnitude of strains applied are generally within the range of those occurring on the surface of long bones, that is, $<2,000 \mu \varepsilon(50)$, where osteoblasts are located (51). On the other hand, bone cell responses to fluid shear stress in vitro do not appear to cause osteoblastic responses. Instead, these studies report increases in bone cell NO and PGE 2 signaling molecule 
release, both of which are thought to be involved in osteocyte mediation of bone remodeling (6, 9). The shear stresses that induce these responses are in the range of those estimated to occur in the canalicular network where osteocytes are embedded $(0.8-3 \mathrm{~Pa}(5))$. Hence, if the stimuli applied in vitro are representative of the in vivo mechanical environment associated with the anatomic location of either an osteoblast or osteocyte (i.e., if the estimates of strain (50) and shear stress are reasonable (5)), the responses induced in our (and others) bone cell cultures are generally responses that have been associated with cells of the osteoblastic or osteocytic phenotype. Furthermore, if the mechanical environment of osteocytes also includes high strains around the lacunae (strains up to $\sim 27,000 \mu \varepsilon$ have been predicted (51)), the experimental reports of increased NO and $\mathrm{PGE}_{2}$ release at higher applied strain as described above $(37,48,49)$ also support this hypothesis.

Although the material properties of adherent cells are time-dependent, in this study a static analysis was considered sufficient to compute the instantaneous structural response of a cell under imposed mechanical loads. Recognizing that certain experimental studies suggest the importance of strain rate (and not merely strain magnitude) in determining cell response (45, 52, 53), a transient viscoelastic analysis would allow investigation of time-dependent changes in the cellular deformations reported here, under pulsating fluid flow and cyclic strain conditions. Our finding that cytoplasm properties have a significant influence on the transmission of deformation to the nucleus is attributed to the large volume of the cytoplasm relative to other components. This indicates the importance of determining precisely the viscoelastic properties and compressibility of the cytoplasm to better understand its role in the structural behavior of adherent cells. The material properties assigned to the cytoskeletal components of the biomechanical model have been used in previous models (28). Similar properties have been estimated theoretically (54) and are in agreement with other experimental work (55). To explain the excessive rigidity of these cytoskeletal components, it has been suggested by Gittes et al. (34) that movement may occur between filaments and tubules. Similarly, in our approach to modeling the structural contribution of cytoskeletal components, relative movement may also occur between interconnected microfilaments and microtubules. However, an improved approach to modeling the cytoskeleton would incorporate a means of transmitting deformation from the membrane to the nucleus, as suggested by Charras and Horton (56). In our modeling approach, a specific tensile or compression-bearing role was not assigned to intermediate filaments because, according to Janmey (46) and Wang and Stamenovic (57), their contribution to cellular rigidity only becomes significant above $20 \%$ strain; their properties were therefore assumed to be incorporated in those of the cytoplasm. Variation in the density of the model mesh (i.e., the number of elements) results in slight variation in the results reported, although the overall differences in deformation due to both fluid shear stress and strain methods remain consistent. The structural behavior of the cell model has been investigated (58), while the quantitative relevance of the model has been confirmed (59), using loading conditions that replicate indentation of an atomic force microscopy probe (60).

In summary, our findings have several implications. The differences in cellular deformation caused by both mechanical stimuli are important for interpretation of cellular responses to mechanical stimuli in vitro, regardless of cell phenotype, and in determining magnitudes of stimuli such that cellular responses could be directly comparable. Although we can conclude that deformations due to both $0.6 \mathrm{~Pa}$ fluid shear stress and 1,000 $\mu \varepsilon$ substrate strain are not equivalent, relating experimentally determined cell responses to fluid shear stress and substrate 
strain treatment to the corresponding predicted deformation patterns suggests a physical basis for bone cell responses to fluid flow and strain stimuli in several studies. Hence, our results highlight the importance of deformation on a cellular level and provide a qualitative and quantitative basis for independent roles for fluid flow and strain in bone physiology.

\section{ACKNOWLEDGMENTS}

Funding was provided by the Program for Research in Third Level Institutions, administered by the Higher Education Authority, and by the BITES (Biomechanical Interactions in Tissue Engineering and Surgical Repair) project funded under the 5th Framework program of the European Commission, QLK3-1999-00559. The authors wish to thank Prof. A. J. El Haj for providing the human bone cells and Dr. Y. Yang and J. Magnay for their assistance in performing the four-point bending apparatus experiments.

\section{REFERENCES}

1. Rubin, C. T., and Lanyon, L. E. (1987) Osteoregulatory nature of mechanical stimuli: function as a determinant for adaptive remodeling in bone. J. Orthop. Res. 5, 300-310

2. Forwood, M. R., and Turner, C. H. (1995) Skeletal adaptations to mechanical usage: results from tibial loading studies in rats. Bone 17, 197S-205S

3. Duncan, R., and Turner, C. H. (1995) Mechanotransduction and the functional response of bone to mechanical strain. Calcif. Tissue Int. 57, 344-358

4. Hillsley, M. V., and Frangos, J. A. (1994) Review: Bone tissue engineering. The role of interstitial fluid flow. Biotechnol. Bioeng. 43, 573-581

5. Weinbaum, S., Cowin, S. C., and Zeng, Y. (1994) A model for the excitation of osteocytes by mechanical loading-induced bone fluid shear stresses. J. Biomech. 27, 339-360

6. Burger, E. H., and Klein-Nulend, J. (1999) Mechanotransduction in bone - role of the lacuno-canalicular network. FASEB J. 13, S101-S112

7. Brown, T. D. (2000) Techniques for mechanical stimulation of cells in vitro: a review. $J$. Biomech. 33, 3-14

8. Hung, C. T., Allen, F. D., Pollack, S. R., and Brighton, C. T. (1996) What is the role of the convective current density in the real-time calcium response of cultured bone cells to fluid flow? J. Biomech. 29, 1403-1409

9. Klein-Nulend, J., van der Plas, A., Semiens, C. M., Ajubi, N. E., Frangos, J. A., Nijweide, P. J., and Burger, E. H. (1995) Sensitivity of osteocytes to biomechanical stress in vitro. FASEB J. 9, 441-445

10. Johnson, D. L., McAllister, T. N., and Frangos, J. A. (1996) Fluid flow stimulates rapid and continuous release of nitric oxide in osteoblasts. Am. J. Physiol. 271, E205-E208 
11. Klein-Nulend, J., Helfrich, M. H., Sterck, J. G., MacPherson, H., Joldersma, M., Ralston, M. H., Semiens, C. M., and Burger, E. H. (1998) Nitric oxide in response to shear stress by human bone cell cultures is endothelial nitric oxide synthase dependent. Biochem. Biophys. Res. Commun. 250, 108-114

12. Bakker, A. D., Soejima, K., Klein-Nulend, J., and Burger, E. H. (2001) The production of nitric oxide and prostaglandin $\mathrm{E}_{2}$ by primary bone cells is shear stress dependent. $J$. Biomech. 34, 671-677

13. Walker, L. M., Publicover, J. M., Preston, M. R., Said Ahmed, M. A. A., and El Haj, A. J. (2000) Calcium channel activation and matrix protein upregulation in bone cells in response to mechanical strain. J. Cell. Biochem. 79, 648-661

14. Jones, D. B., Nolte, H., Scholubbers, J. G., Turner, E., and Veltel, D. (1991) Biochemical signal transduction of mechanical strain in osteoblast-like cells. Biomaterials 12, 101-110

15. Kaspar, D., Seidl, W., Neidlinger-Wilke, C., Ignatius, A., and Claes, L. (2000) Dynamic cell stretching increases human osteoblast proliferation but decreases osteocalcin synthesis and alkaline phosphotase activity. J. Biomech. 33, 45-51

16. Peake, M. A., Cooling, L. M., Magnay, J. L., Thomas, P. B. M., and El Haj, A. J. (2000) Cellular responses to mechanical stress. Selected contribution: regulatory pathways involved in mechanical induction of $c$-fos gene expression in bone cells. J. Appl. Physiol. 89, 24982507

17. Owan, I., Burr, D. B., Turner, C. H., Qui, J., Tu, Y., Onyia, J. E., and Duncan, R. L. (1997) Mechanotransduction in bone: osteoblasts are more responsive to fluid forces than mechanical strain. Am. J. Physiol. 273, C810-C815

18. Smalt, R., Mitchell, F. T., Howard, R. L., and Chambers, T. J. (1997) Induction of NO and prostaglandin $\mathrm{E}_{2}$ in osteoblasts by wall-shear stress but not mechanical strain. Am. J. Physiol. 273, E751-E758

19. You, J., Yellowley, C. E., Donahue, H. J., Zhang, Y., Chen, Q., and Jacobs, C. R. (2000) Substrate deformation levels associated with routine physical activity are less stimulatory to bone cells relative to loading-induced oscillatory fluid flow. J. Biomech. Eng. 122, 387-393

20. Evans, E., and Yeung, A. (1989) Apparent viscosity and cortical tension of blood granulocytes determined by micropipette aspiration. Biophys. J. 56, 151-160

21. Guilak, F., and Mow, C. (2000) The mechanical environment of the chondrocyte: a biphasic finite element model of cell-matrix interactions in articular cartilage. J. Biomech. 33, 16631673

22. Kamm, R. D., McVittie, A. K., and Bathe, M. (2000) On the role of continuum models in mechanobiology. ASME International Congress - Mechanics in Biology 242, 1-9. 
23. Karcher, H., Lammerding, J., Huang, H., Lee, R. T., Kamm, R. D., and Kaazempur, M. R. (2003) A three-dimensional viscoelastic model for cell deformation with experimental verification. Biophys. J. 85, 3336-3349

24. Wang, N., Butler, J. P., and Ingber, D. E. (1993) Mechanotransduction across the cell surface and through the cytoskeleton. Science 26, 1124-1127

25. Heidemann, S. R., Kaech, S., Buxbaum, R. E., and Matus, A. (1999) Direct observations of the mechanical behaviors of the cytoskeleton in living fibroblasts. J. Cell Biol. 145, 109-122

26. Shafrir, Y. G., and Forgacs, G. (2002) Mechanotransduction through the cytoskeleton. Am. J. Physiol. 282, C479-C486

27. Ingber, D. E. (1997) Tensegrity: the architectural basis of cellular mechanotransduction. Annu. Rev. Physiol. 59, 575-599

28. Stamenovic, D., Fredberg, J. J., Wang, N., Butler, J., and Ingber, D. E. (1996) A microstructural approach to cytoskeletal mechanics based on tensegrity. J. Theor. Biol. 181, 125-136

29. Wang, N., Naruse, K., and Stamenovic, D. (2001) Mechanical behavior in living cells consistent with the tensegrity model. Proc. Natl. Acad. Sci. USA 98, 7765-7770

30. Prendergast, P. J. (1997) Finite element analysis in tissue mechanics and orthopaedic implant design. Clin. Biomech. 12, 343-368

31. Frisch, T., and Thoumine, O. (2002) Predicting the kinetics of cell spreading. J. Biomech. 35, 1137-1141

32. Guilak, F., Tedrow, J. R., and Burgkart, R. (2000) Viscoelastic properties of the cell nucleus. Biochem. Biophys. Res. Commun. 269, 781-786

33. Shin, D., and Athanasiou, K. (1999) Cytoindentation for obtaining cell biomechanical properties. J. Orthop. Res. 17, 880-890

34. Gittes, F., Mickey, B., Nettleton, J., and Howard, J. (1993) Flexural rigidity of microtubules and actin filaments measured from thermal fluctuations in shape. J. Cell Biol. 120, 923-934

35. Ajubi, N. E., Klein-Nulend, J., Nijweide, P. J., Vrijheid-Lammers, T., Alblas, M. J., and Burger, E. H. (1996) Pulsating fluid flow increases prostaglandin by cultured chicken osteocytes: a cytoskeleton dependent process. Biochem. Biophys. Res. Commun. 225, 62-68

36. Pavalko, F. M., Chen, N. X., Turner, C. H., Burr, D. B., Atkinson, S., Hsieh, Y.-F., Qui, J., and Duncan, R. L. (1998) Fluid shear-induced mechanical signaling in MC3T3-E1 osteoblasts requires cytoskeleton-integrin interactions. Am. J. Physiol. 275, C1591-C1601

37. Zaman, G., Pitsillides, A. A., Rawlinson, S. C. F., Suswillo, R. F. L., Mosley, J. R., Cheng, M. Z., Platts, L. A. M., Hukkanen, M., Polak, J. M., and Lanyon, L. E. (1999) Mechanical 
strain stimulates nitric oxide production by rapid activation of endothelial nitric oxide synthase in osteocytes. J. Bone Miner. Res. 14, 1123-1131

38. Helfrich, M. H., Evans, D. E., Grabowski, P. S., Pollock, J. S., Ohshima, H., and Ralston, M. H. (1997) Expression of nitric oxide synthase isoforms in bone and bone cell cultures. $J$. Bone Miner. Res. 12, 1108-1115

39. Turner, C. H., Takano, Y., Owan, I., and Murrell, G. A. (1996) Nitric oxide inhibitor LNAME suppresses mechanically induced bone formation in rats. Am. J. Physiol. 270, E634E639

40. Fox, S. W., Chambers, T. J., and Chow, J. W. (1996) Nitric oxide is an early mediator of the increase in bone formation by mechanical stimulation. Am. J. Physiol. 270, E955-E960

41. Forwood, M. R. (1996) Inducible cyclooxygenase (COX-2) mediates the induction of bone formation by mechanical loading in vivo. J. Bone Miner. Res. II, 1688-1693

42. Chambers, T. J., Chow, J. W., Fox, S. W., Jagger, C. J., and Lean, J. M. (1997) The role of prostaglandins and nitric oxide in the response of bone to mechanical stimulation. Adv. Exp. Med. Biol. 433, 295-298

43. Chow, J. W. M., Jagger, C. J., and Chambers, T. J. (1993) Indomethacin has distinct early and late actions on bone formation induced by mechanical stimulation. Am. J. Physiol. 265, E340-E347

44. Pead, M. J., and Lanyon, L. E. (1989) Indomethacin modulation of load-related stimulation of new bone formation in vivo. Calcif. Tissue Int. 45, 34-40

45. Bacabac, R. G., Smit, T. H., Mullender, M. G., Dijcks, S. J., Van Loon, J. J. W. A., and Klein-Nulend, J. (2004) Nitric oxide production by bone cells is fluid shear stress rate dependent. Biochem. Biophys. Res. Commun. 315, 823-829

46. Janmey, P. A. (1991) The cytoskeleton and cell signaling: component localisation and mechanical coupling. Physiol. Rev. 78, 763-781

47. Dong, C., and Lei X. X. (2000) Biomechanics of cell rolling: shear flow, cell-surface adhesion, and cell deformability. J. Biomech. 33, 1, 35-43.

48. Pitsillides, A. A., Rawlinson, S. C., Suswillo, R. F., Bourrin, S., Zaman, G., and Lanyon, L. E. (1995) Mechanical strain-induced NO production by bone cells: a possible role in adaptive bone (re)modeling? FASEB J. 9, 1614-1622

49. Fermor, B., Gundle, R., Evans, M., Emerton, M., Pocock, A., and Murray, D. (1998) Primary human osteoblast proliferation and prostaglandin release in response to mechanical strain in vitro. Bone 22, 637-643 
50. Burr, D. B., Milgrom, C., Fyhrie, D., Forwood, M., Nyska, M., Finestone, A., Hoshaw, S., Saiag, E., and Simkin, A. (1996) In vivo measurement of human tibial strains during vigorous activity. Bone 18, 405-410

51. McCreadie, B. R., and Hollister, S. J. (1997) Strain concentrations surrounding an ellipsoid model of lacunae and osteocytes. Comput. Methods Biomech. Biomed. Eng. 1, 61-68

52. Turner, C. H., Owan, I., and Takano, Y. (1995) Mechanotransduction in bone: role of strain rate. Am. J. Physiol. 269, E438-E442

53. Mosley, J. R., and Lanyon, L. E. (1998) Strain rate as a controlling influence on adaptive modeling in response to dynamic loading of the ulna in growing male rat. Bone 23, 313-318

54. Ben-Avraham, D., and Tirion, M. M. (1995) Dynamic and elastic properties of F-actin: a normal modes analysis. Biophys. J. 68, 1231-1245

55. Tsuda, Y., Yasutake, H., Ishijima, A., and Yanagida, T. (1996) Torsional rigidity of single actin filaments and actin bond breaking force under torsion measured directly by in vitro micromanipulation. Proc. Natl. Acad. Sci. USA 93, 12,937-12,942

56. Charras, G. T., and Horton, M. A. (2002) Single cell mechanotransduction and its modulation analyzed by atomic force microscopy indentation. Biophys. J. 82, 2970-2981

57. Wang, N., and Stamenovic, D. (2000) Contribution of intermediate filaments to cell stiffness, stiffening and growth. Am. J. Physiol. 279, C188-C194

58. McGarry, J. G., and Prendergast, P. J. (2004) A three-dimensional finite element model of an adherent eukaryotic cell. Eur. Cell Mater. 7, 27-34

59. McGarry, J. G., and Prendergast, P. J. (2003) A finite element model of an adherent cell: comparison of simulated cell indentation with atomic force microscopy experiments. Proceedings of the International Congress on Computational Bioengineering 2, 552-559.

60. Mathur, A. B., Collinsworth, A. M., Reichert, W. M., Kraus, W. E., and Truskey, G. A. (2001) Endothelial, cardiac muscle and skeletal muscle exhibit different viscous and elastic properties as determined by atomic force microscopy. J. Biomech. 34, 1545-1553

Received May 15, 2004; accepted November 9, 2004. 
Table 1

Material properties assigned to components of the cell model

\begin{tabular}{|l|l|l|}
\hline Model Component & Elastic modulus, Pa & Poisson's ratio,v \\
\hline Cytoplasm & $100(22)$ & $0.37(33)$ \\
\hline Nucleus & $400(32)$ & $0.37(33)$ \\
\hline Membrane (22) & 103 & 0.3 \\
\hline Microtubules (34) & $1.2 \times 10^{9}$ & 0.3 \\
\hline Microfilaments (34) & $2.6 \times 10^{9}$ & 0.3 \\
\hline
\end{tabular}


Fig. 1

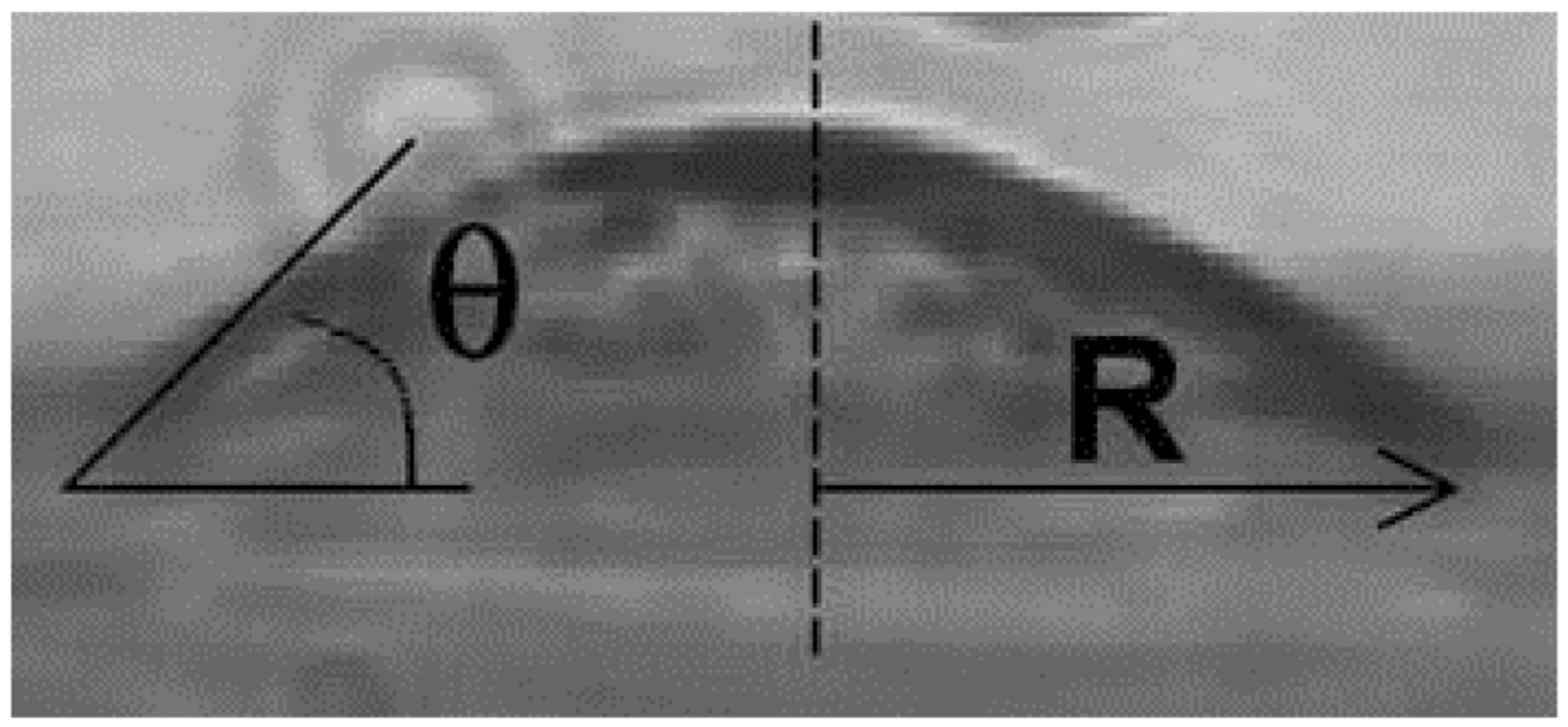

Figure 1. Image of a spreading chick embryo fibroblast $3 \mathrm{~h}$ after plating on a glass microplate. The dimensions of the cell model are based on the highlighted radius $(\mathrm{R})$ and contact angle $(\theta)$, while the dashed line represents the central axis of the model geometry. Adapted with permission from Frisch and Thoumine (39). 
Fig. 2

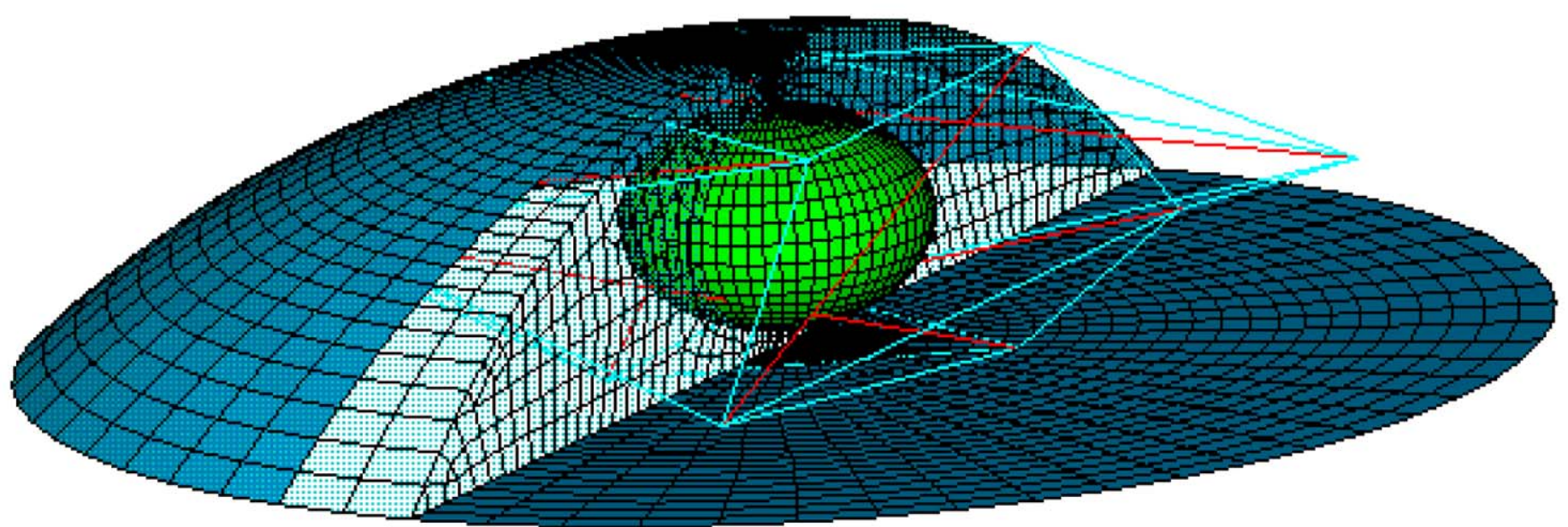

Figure 2. Three-dimensional computational biomechanical model of an adherent cell. The cell model comprises nucleus (in green), microfilament (blue lines), microtubule (red lines), cytoplasm (internal transparent elements), and membrane (dark blue) components. Half of the cytoplasm and membrane components are omitted for clarity. Cell model dimensions (contact radius, $19.2 \mu \mathrm{m}$; height, $7.6 \mu \mathrm{m}$ ) are based on the radius $(\mathrm{R})$ and contact angle $(\theta)$ highlighted in Fig. 1 and model volume is $\sim 3,000 \mu \mathrm{m}^{3}$. 
Fig. 3

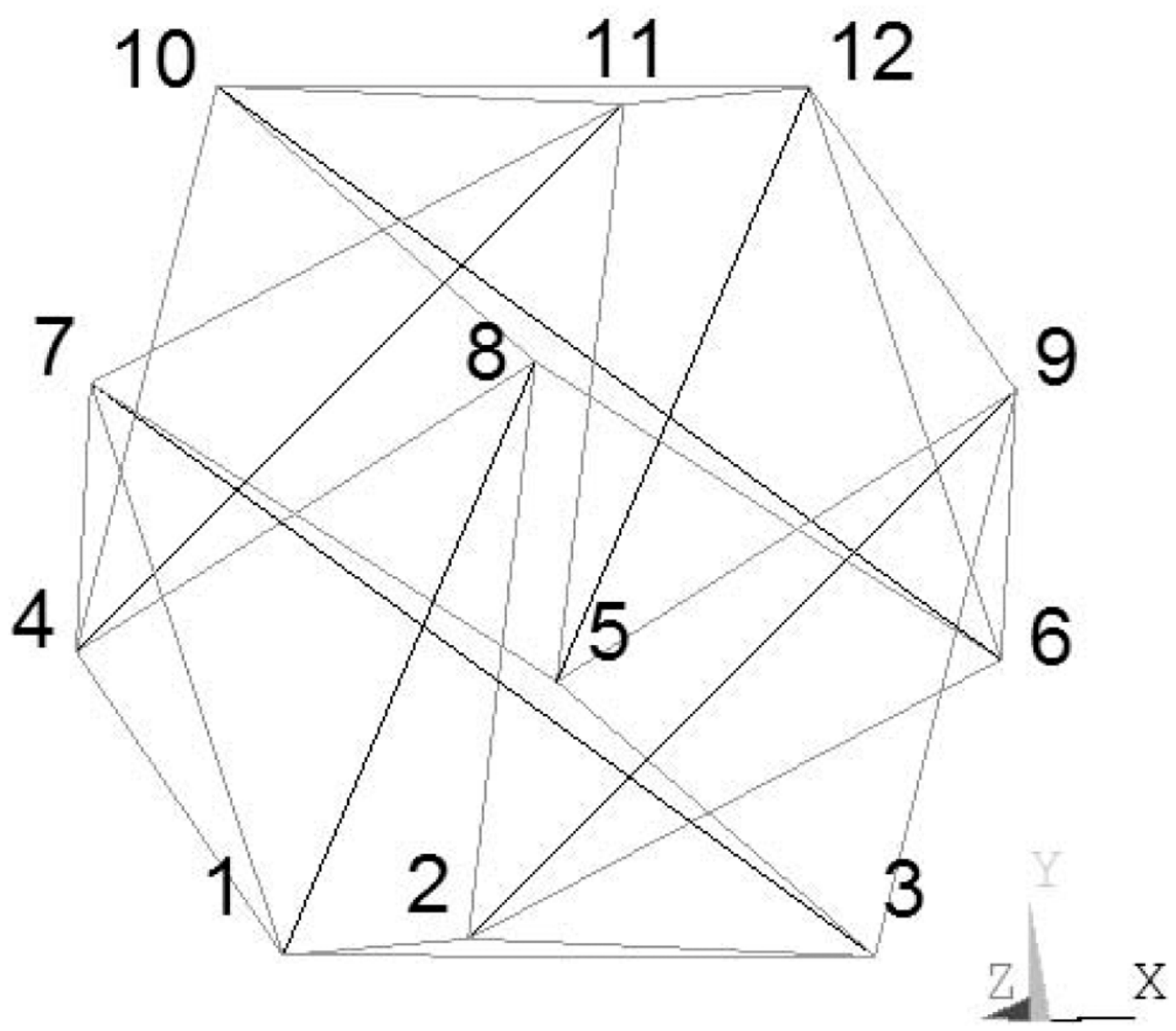

Figure 3. A three-dimensional tensegrity network of six compression-bearing struts (two in each orthogonal direction), representing the aggregate behavior of microtubules (dark lines), surrounded by and connected with 24 tensional cables, representing the aggregate behavior of microfilaments (light lines). The internal cytoskeleton in the cell model (Fig. 2) is arrived at by altering the lengths and positioning of the microtubule and microfilament components in order to fit the cytoskeletal network to a wider spread configuration (Fig. 2). See Materials and Methods for further explanation. 
Fig. 4

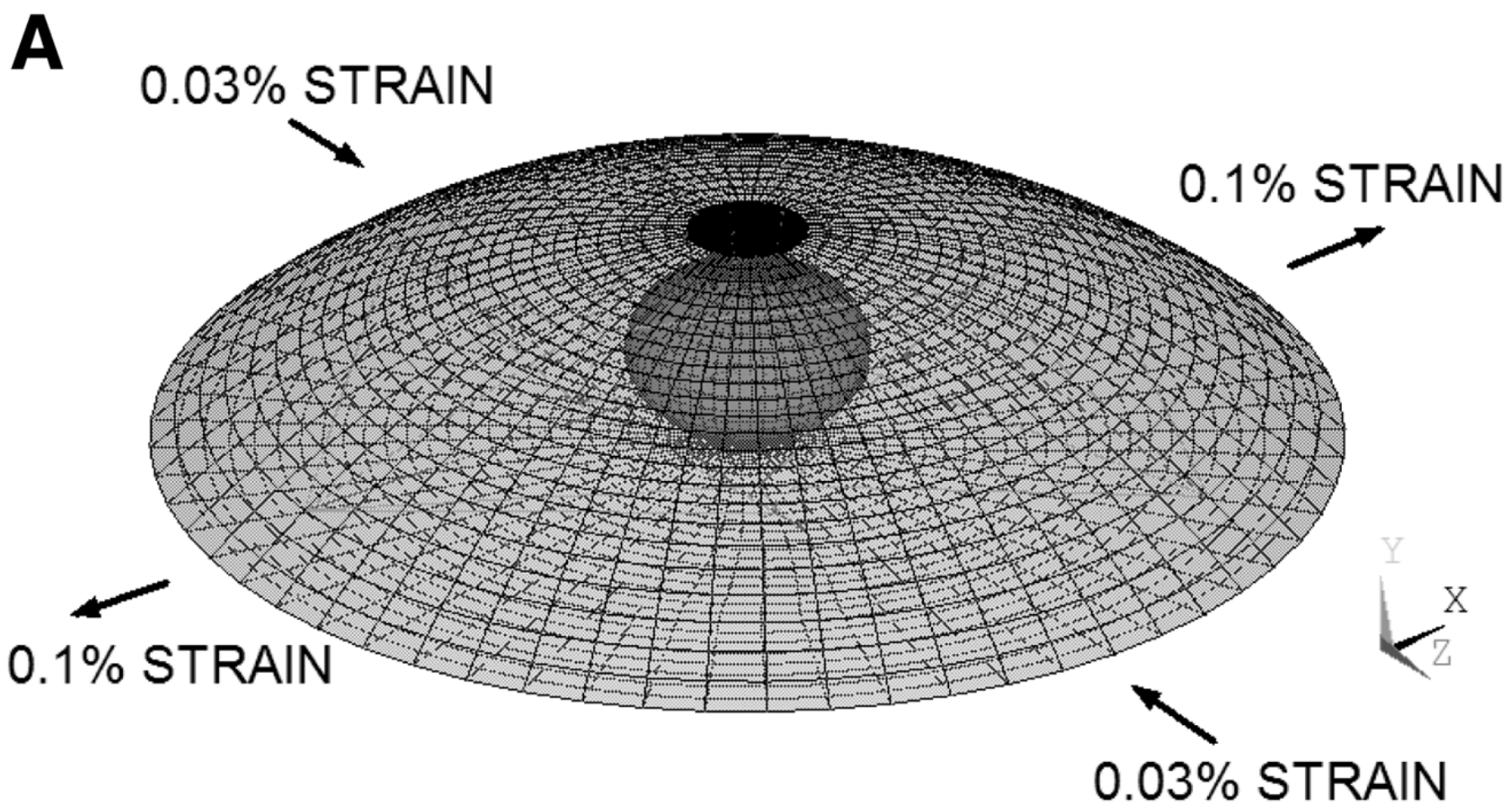

B

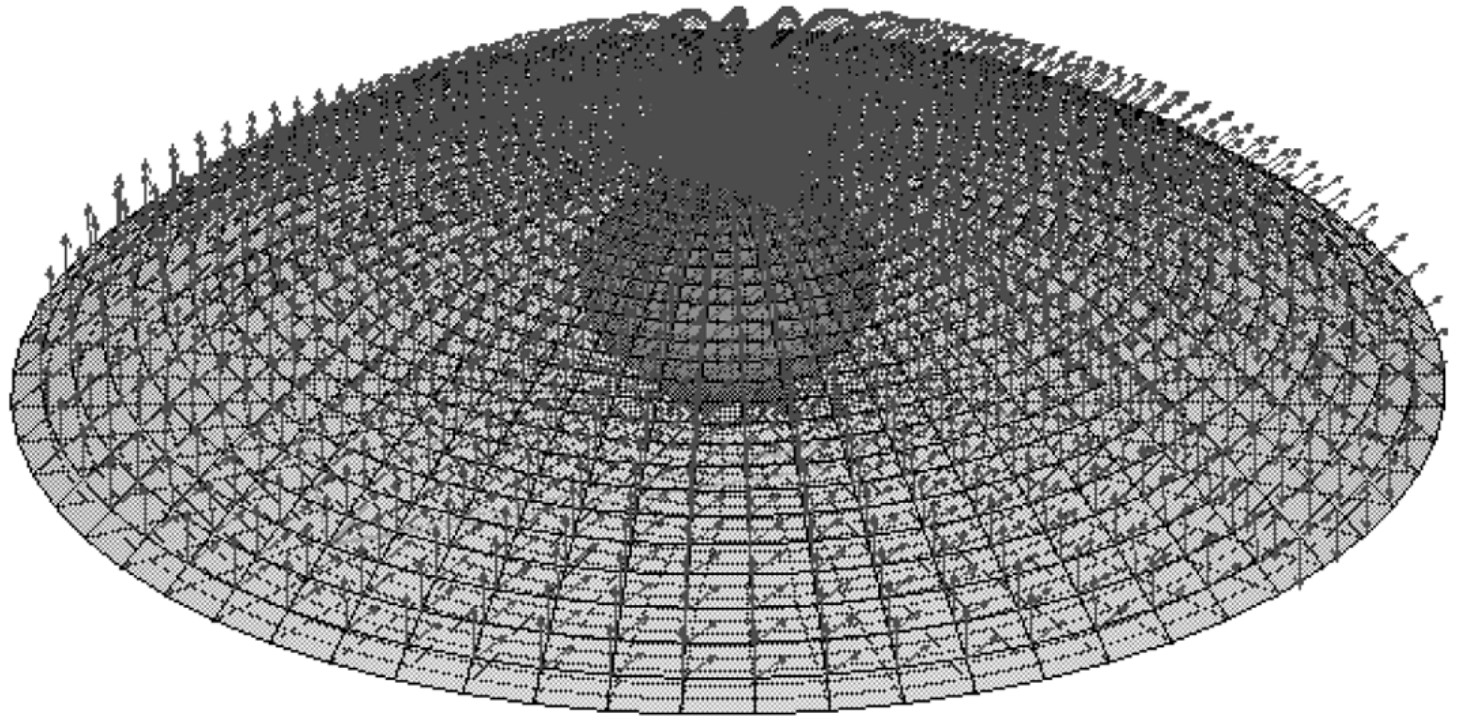

Figure 4. Loading conditions applied to the cell model in order to model the effect of substrate strain and fluid shear stress on a single adherent bone cell. To model substrate strain, nodes at the at the cell-substrate interface (see text) are constrained in the $y$ (vertical) direction, and displaced by $0.1 \%$ and $0.03 \%$ strain in the $x$ and $z$ directions, respectively $(\boldsymbol{A})$. To model fluid flow in the $x$ direction, tangential forces (resolved to $x$ and $y$ components) are applied to each membrane surface node (arrows in $\boldsymbol{A}$ ), based on the area of each surrounding membrane elements $(\boldsymbol{B})$. See Materials and Methods for further explanation. 
Fig. 5
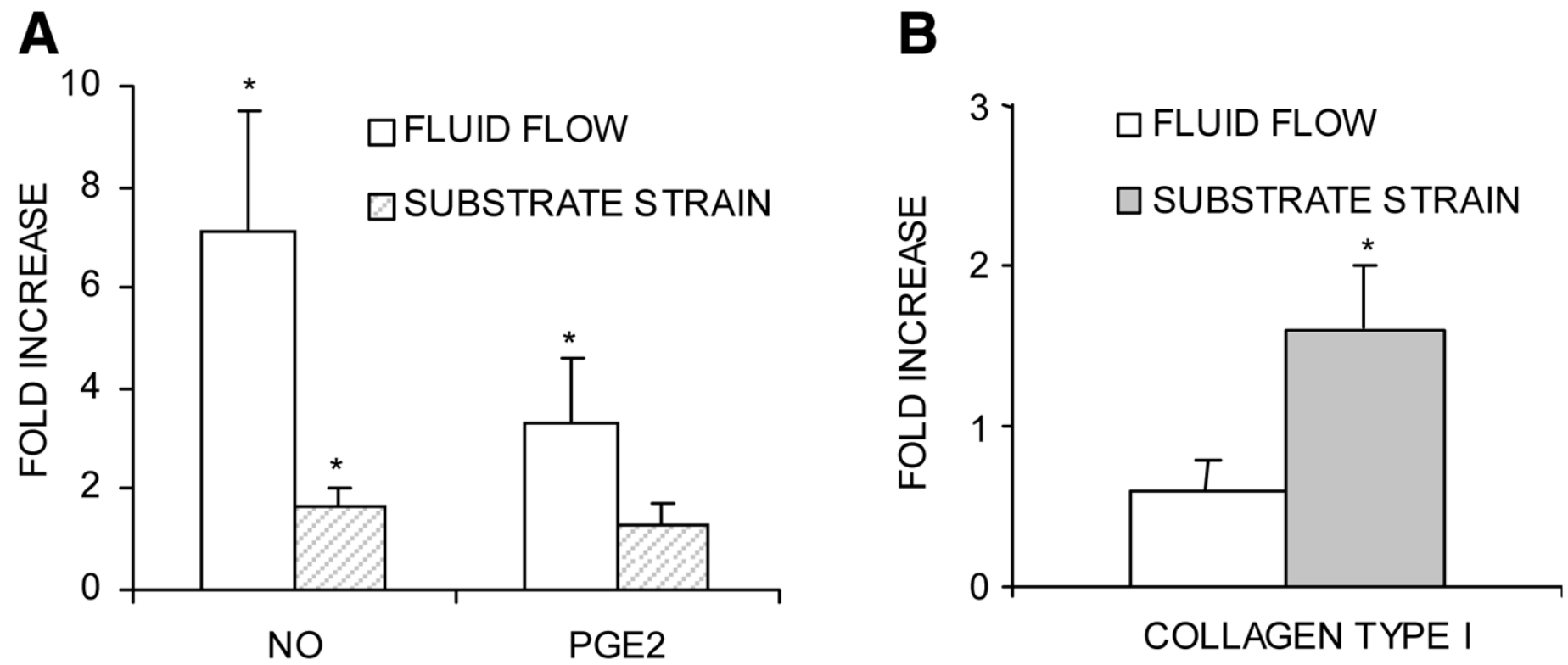

Figure 5. Effect of cyclic substrate strain and pulsatile fluid flow on prostaglandin $\mathrm{E}_{2}\left(\mathrm{PGE}_{2}\right)$, nitric oxide (NO), and collagen type I production by human bone cells in vitro. All values are expressed as treatment-control ratios (fold increase) of the mean values $\pm \mathrm{SEM}$. Both $\mathrm{NO}$ and $\mathrm{PGE}_{2}$ release were significantly increased after fluid shear stress treatment for $60 \mathrm{~min}(\boldsymbol{A})$. Production of collagen type I was increased by strain but decreased by fluid shear stress treatment, both applied for $60 \mathrm{~min}$ followed by $24 \mathrm{~h}$ postincubation $(\boldsymbol{B})$. *Fold increase value differs significantly from 1 $(P<0.05)$. 
Fig. 6
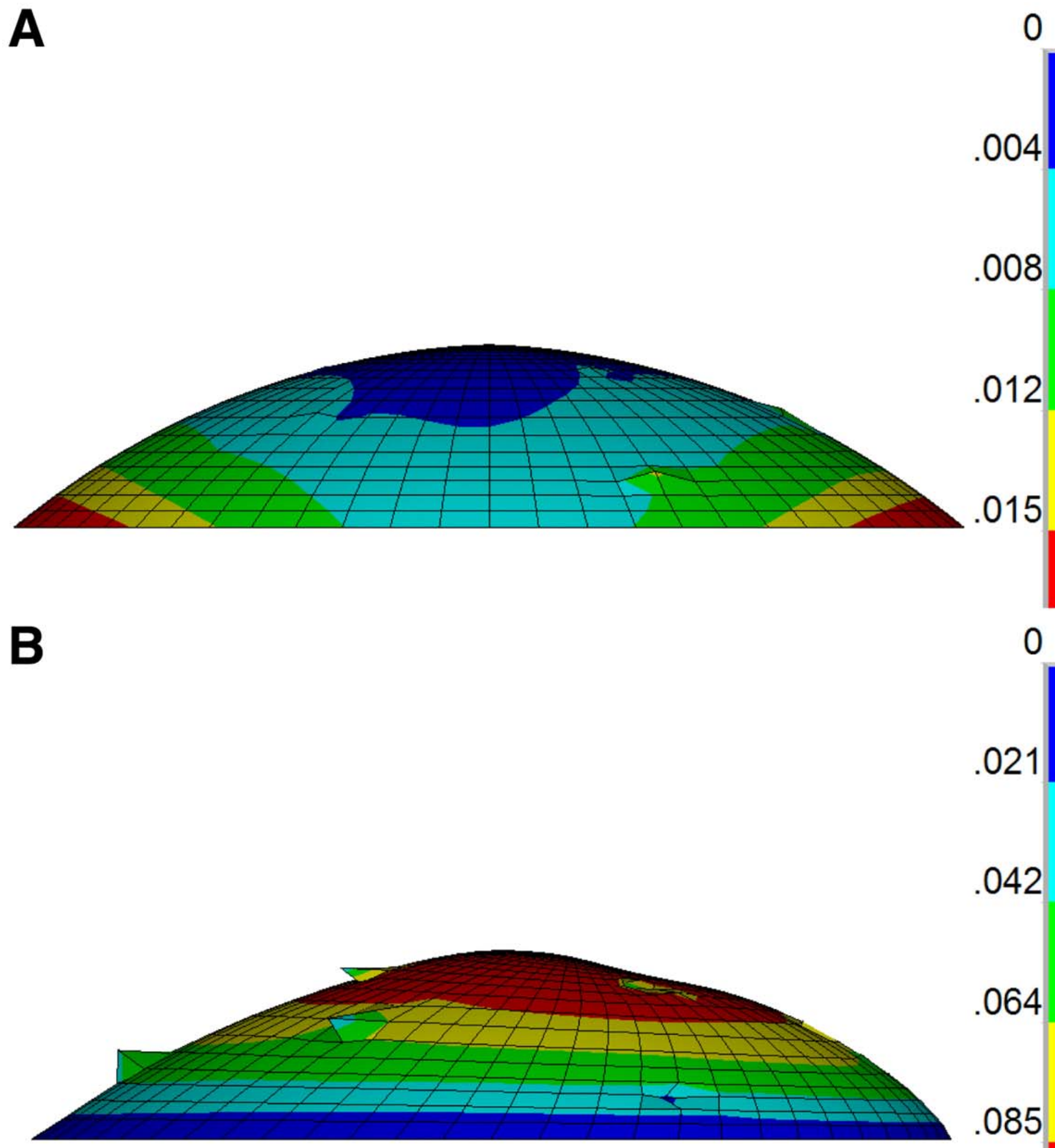

Figure 6. Comparison of displacements in the cell model due to both substrate strain and fluid shear stress loading conditions. The contour legend indicates the magnitude of displacement $(\mu \mathrm{m})$ due to substrate strain $(\boldsymbol{A})$ and fluid shear stress $(\boldsymbol{B})$. The image is exaggerated 30 -fold to illustrate the differences in deformation. Note the resistance to displacement at receptor sites, where the cytoskeleton merges with the membrane. 
Fig. 7

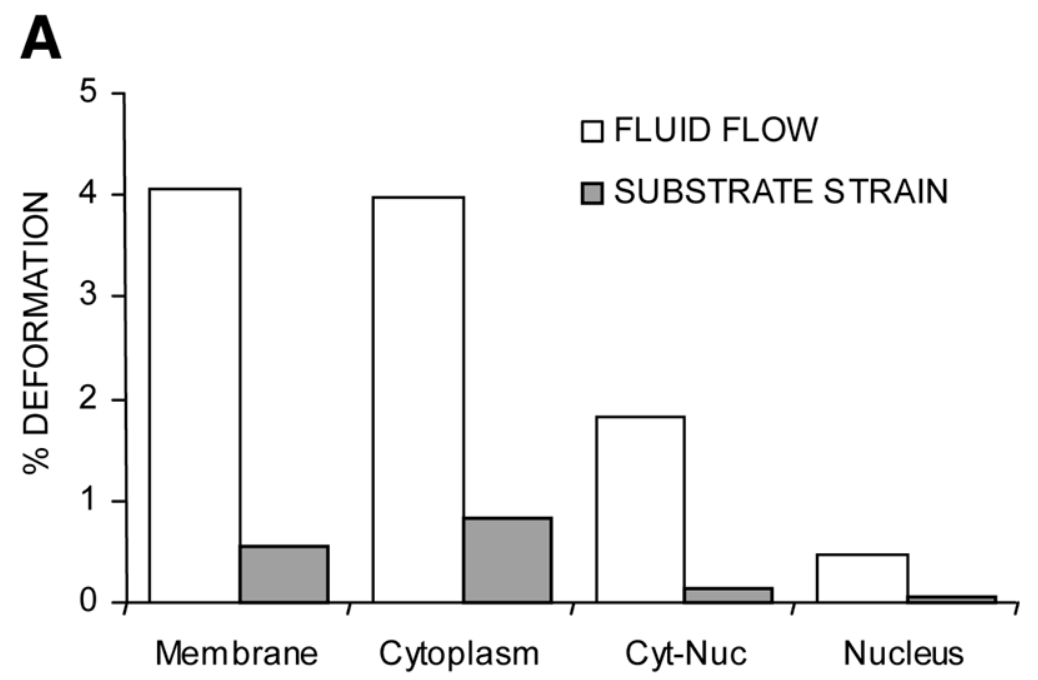

B

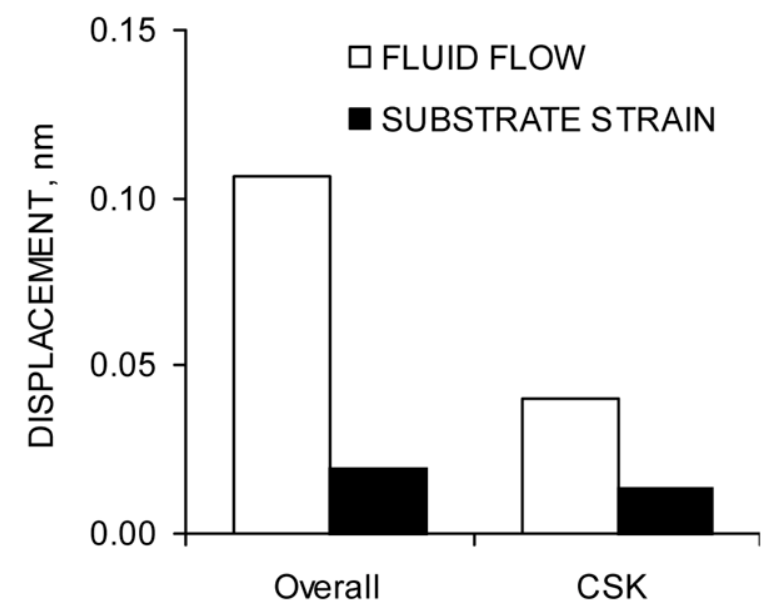

Figure 7. Overview of the differences in deformation and displacement of cell model components caused by both fluid shear stress and substrate strain loading conditions. Deformation is expressed as the maximum von Mises stress as a percentage of original elastic properties for each model component $(\boldsymbol{A})$. Membrane and cytoskeleton displacements $(\mu \mathrm{m})$ are also maximum values $(\boldsymbol{B})$. Cyt-Nuc, the region of the cytoplasm immediately surrounding the nucleus; CSK, cytoskeleton. 